\title{
EL VIBRACIONISMO DE RAFAEL BARRADAS: GENEALOGÍA DE UN CONCEPTO
}

\author{
$M^{a}$ LlUIISA FAXEDAs BRUJATS \\ Universitat de Girona
}

\begin{abstract}
El vibracionismo fue el movimiento pictórico practicado por el pintor Rafael Barradas (1890-1929) entre 1917 y 1920, y constituye una de las etapas artísticas más reconocidas y reconocibles de su trayectoría. El objetivo del artículo es el de profundizar en el concepto mismo de "vibracionismo", para descubrir qué elementos de su propuesta plástica pretendía Barradas poner de relieve al escoger esta palabra en particular. Partiremos del frecuente uso del concepto de vibración en los medios de la vanguardia europea, para centrarnos después en su aparición en relación a la obra barradiana a partir de 1917; finalmente se analiza su significado a partir de un texto publicado por Torres-García, y se estudia su concreción plástica en la obra pictórica del autor.

Palabras clave: Vibracionismo; Rafael Barradas; Joaquín Torres García; Joan Salvat Papasseit; Vanguardia en España.
\end{abstract}

\section{THE VIBRATIONISM OF RAFAEL BARRADAS: GENEALOGY OF A CONCEPT}

Vibrationism was an art movement developed by the painter Rafael Barradas (1890-1929) between 1917 and 1920, and this period remains one of the most recognized and recognizable of his artistic legacy. The aim of this paper is to look further into the concept of vibrationism in an attempt to reveal which elements of Barradas' artistic vision he sought to highlight by choosing this particular word. The author begins by addressing the frequent use of the concept of vibration in European avant-garde circles and then focuses on its emergence in Barradas' works from 1917 onwards. Finally, the meaning given to vibrationism in a text published by Torres-García is analyzed and his artistic interpretation of the style in his own paintings is discussed.

Key words: Vibrationism; Rafael Barradas; Joaquín Torres García; Joan Salvat Papasseit; Spanish vanguard.

El vibracionismo, primero de los "ismos" propuestos por el pintor uruguayo Rafael Barradas (1890-1929), ha sido generalmente considerado como el resultado de su síntesis personal del futurismo, el simultaneísmo, y en menor grado el cubismo. Este artículo, más que discutir las fuentes plásticas del movimiento, pretende profundizar en el concepto mismo de "vibracionismo", investigando sus precedentes y planteando qué elementos de su propuesta plástica quiso Barradas porner de relieve al escoger este término en particular.

"Sans vibration il n'y a pas de vie" 
A finales del XIX culminó el desarrollo de una teoría científica de las vibraciones que consideraba que la luz, al igual que el sonido, era un fenómeno de naturaleza vibratoria'; así, las diferencias entre los colores dependerían de las distintas frecuencias de las vibraciones con las que la luz excita la retina, de la misma forma que los sonidos son producidos por las distintas vibraciones del aire. La idea de que color y sonido estaban compuestos por vibraciones similares de altura y velocidad distintas ya había sido propuesta por el matemático Leonhard Euler en 1761, y confirmada por el científico Thomas Young, quien dio a esta teoría de base analógica su formulación moderna demostrando la naturaleza ondulatoria de la luz, a principios del s. XIX ${ }^{2}$. Obviamente todo lo relacionado con las características de la luz interesaba particularmente a los pintores, quiénes se informaban mediante diversas fuentes; así Charles Blanc incluyó en su influyente Grammaire des arts du dessin (1876) un capítulo titulado "La vibration des couleurs" en qué enuncia: "De même que le grave ou l'aigu des sons dépendent du nombre des vibrations que rend la corde tendue, dans un temps donné, de même on peut dire que chaque couleur est astreiente à un certain nombre de vibrations qui agissent sur l'organe de la vue comme les sons sur l'organe de l'ouïe"3.

Estas investigaciones resultaron de especial interés para aquellos pintores que, ya desde finales del s. XIX, desarrollaron en su obra una evolución que acabaría culminando en lo que conocemos como pintura abstracta. Así Gauguin escribió lo siguiente: "Pensez aussi à la part musicale que prendra désormais la couleur dans la peinture moderne. La couleur qui est vibration de même que la musique est à même d'atteindre ce qu'il y a de plus général et partant de plus vague dans la nature: sa force intérieure" ". Otros artistas que también se interesaron por la cuestión fueron los neoimpresionistas, Georges Vantongerloo o Robert Delaunay, para quién la cuestión de las relaciones y diferencias entre los colores se planteaba precisamente en términos de vibraciones: "[...] la couleur étant une mesure en vibration selon telle ou telle intensité, vu son voisinage et vu sa superficie, dans ses rapports avec tous les couleurs. Telle vibration d'un orangé placé dans la composition à côté d'un jaune - ces deux couleurs étant placées sur le diagramme des couleurs presque côte à côte, leurs vibrations étant par conséquent voisines, vibrent très vite. $\mathrm{Si}$, dans la composition il y a un bleu violet, le bleu violet formera une vibration avec l'orange jaune: un mouvement beaucoup plus lent"s.

Si la de Delaunay era una posición estrictamente científica, otros autores se interesaron por la historia filosófica del mismo concepto de vibración, poniéndola en relación con la utopía de la obra de arte total, y por extensión con los debates sobre el ideal de la unidad del mundo ${ }^{6}$. Según esta línea de pensamiento, de origen platónico, las vibraciones no sólo explicarían las analogías entre luz y sonido, sino también las relaciones entre los diversos niveles de la existencia, como escribió Plotino: "[...] así que el influjo correspondiente a la plegaria se debe a que una parte entró en simpatía con otra, como ocurre en una misma cuerda cuando está tensada: al vibrar por la parte de abajo, vibra también arriba [...] Más si la vibración de una lira repercute incluso en otra según el alcance de la simpatía, entonces también en el universo habrá una sola armonía aunque provenga de contrarios [...]"’.

El de la vibración es pues un fenómeno intangible e invisible, que permite sin embargo conectar elementos distintos entre sí; la unidad vibratoria esencial entre el sonido y el color descubierta por los científicos apoyaría la idea de la existencia de una harmonía universal de naturaleza esencialmente vibratoria. La fuerza de esta idea hizo del de "vibración" un término muy usado en la teoría artística finisecular, especialmente en el entorno simbolista por autores como Baudelaire o Ma-

\footnotetext{
${ }^{1}$ Roque, 2002.

2 Roque, 2003: 52-53. Gamwell, 2002: 152.

3 Blanc, 1876: 569-570.

4 Gauguin, 1974: 222.

${ }^{5}$ Delaunay, 1957: 60-61.

${ }^{6}$ Lista, 2006.

Plotino, Enéada IV, 4, 41, 1-9.
} 
llarmé, entre otros; una de sus concreciones en el ámbito artístico fue el auge de las sinestesias, especialmente de la audition colorée, como forma artística que adquiriría una importancia central ${ }^{8}$. Henri Rovel publicó en la revista Les Tendances Nouvelles un artículo titulado "Les lois d'harmonie de la Peinture et de la Musique sont les mêmes" (1908) en qué unía el aspecto sinestético y el espiritual implícitos, según él, en la teoría de las vibraciones: "L'être humain est un; toutes les sensations d'harmonie qu'il éprouve sont les résultats des vibrations; par conséquent, qu'il perçoive ces sensations par les yeux ou par les oreilles, les lois qui les régissent doivent être les mêmes. [...] La vie est caractérisée par la vibration. Sans vibration il n'y a pas de vie"

Estas ideas influyeron poderosamente en algunos artistas vinculados a los círculos simbolistas, muy interesados por la dimensión trascendental de su trabajo; Vassily Kandinsky, por ejemplo, utilizó la teoría de las vibraciones para explicar cómo el contenido espiritual de la obra de arte entra en contacto con el alma del espectador: "The inner element, taken in isolation, is the emotion in the soul of the artist that causes a corresponding vibration [...] in the soul of another person, the receiver" ${ }^{\prime \prime}$. Si todos los objetos, colores y formas emiten vibraciones o resonancias que el alma puede captar, las vibraciones se convierten en el contenido espiritual del arte; el objetivo del artista será hacer que su obra sea capaz de transmitir este contenido de la forma más directa posible, lo que finalmente le hará liberarse del objeto. En la misma línea, el compositor Alexander Scriabin señaló que las vibraciones permiten entender la continuidad entre los diversos estados de conciencia, y entre estos y el mundo ${ }^{11}$; y el pintor Frantisek Kupka escribió sobre las vibraciones como un elemento clave en las relaciones entre colores y sentimientos, y en la relación de los colores entre si $^{12}$.

Para muchos de estos autores el concepto de vibración sirvió pues para expresar un auténtico "monismo de las sensaciones"13, es decir, una unidad entre lo que perciben los distintos sentidos entre sí, y entre el sujeto perceptor y lo percibido; es sobre todo en este sentido que el término de vibración aparecerá repetidamente en la literatura futurista. La palabra ya aparece en el Manifiesto futurista de Marinetti (1909) $)^{14}$, quién escribe en "Parole in libertà" (1914): "Unica preoccupazione del narratore rendere tutte le vibrazioni del suo io. Se questo narratore dotato di lirismo avrà inoltre una mente popolata di idee generali, involontariamente allacerà le sue sensazioni coll'universo intero sconosciuto o intuito da lui" ${ }^{\prime 5}$. Las vibraciones son pues el elemento invisible que conecta sus ideas y emociones con las del universo que le rodea en una relación completamente irracional, y por ello propone eliminar toda noción de sintaxis, puntuación o articulación del texto. Encontramos referencias a las vibraciones en otros documentos y obras futuristas ${ }^{16}$, y especialmente en el texto de Carlo Carrá La peinture de sons, bruits et odeurs (1913): "Il est indiscutable: $1^{\circ}$ que le silence est statique et que les sons, bruits et odeurs sont dynamiques; $2^{\circ}$ que les sons, bruits et odeurs sont des formes et des intensités différentes de vibration; $3^{\circ}$ que chaque succession de sons, bruits et odeurs imprime dans l'esprit une arabesque de formes et des couleurs" ${ }^{\prime 17}$. Carrá defiende una pintura multisensorial que reúna la riqueza de sensaciones que ofrece la vida moderna al artista; por ello plantea que en los cuadros se expresen las equivalencias plásticas de los sonidos, ruidos y

\footnotetext{
${ }^{8}$ Faxedas, 2015.

${ }^{9}$ Rovel, 1908: p. 721.

${ }^{10}$ Lindsay-Vergo, 1994: 87.

${ }^{11}$ Scriabin, 1979: 67-68.

${ }^{12}$ Kupka, 1989: 187.

${ }^{13}$ Roque, 2003: 54.

${ }^{14}$ Marinetti, 1996: 11.

15 Marinetti, 1996: 70.

${ }^{16}$ Una de las pinturas de Giacomo Balla de 1913-14 lleva por subtítulo Vibraciones prismáticas.

${ }_{17}$ Texto publicado originalmente en italiano en el no 17 de la revista Lacerba (Florencia) (1-IX-1913). Ver Lista, 1973: 182-186; 183.
}

Arch. esp. arte, LXXXVIII, 351, JULIO-SEPTIEMBRE 2015, 281-298

ISSN: 0004-0428, eISSN: 1988-8511, doi: 10.3989/aearte.2015.17 
olores de teatros, cines, puertos o garajes, como habían intentado los pintores futuristas ${ }^{18}$, evitando toda referencia trascendental y proponiendo la abolición de toda interpretación racional del mundo.

En resumen, el concepto de vibración se utilizó en las vanguardias europeas con diversos sentidos relacionados entre sí: en primer lugar para referirse a la fenomenología física de la luz y a la supuesta vibración de los colores; en segundo lugar para establecer un campo de comparación entre luz y sonido, lo que sería el punto de partida para todo tipo de investigaciones sinestéticas; y finalmente para referirse a cierta conexión o unidad emocional entre el sujeto y su entorno. Ecos de los tres aspectos los encontraremos en la obra barradiana, como veremos.

\section{Los orígenes del vibracionismo}

La cronología del vibracionismo no ha sido establecida con claridad debido a la falta de datos y al hecho de que Barradas no dejó ningún testimonio sobre el mismo. Podemos afirmar que la estética y la plástica vibracionistas se desarrollaron en su pintura a lo largo de 1917, antes incluso de que él mismo acuñara el término ${ }^{19}$. Barradas volvió a Barcelona a finales de febrero o principios de marzo de 1916, después de una estancia en Zaragoza. En julio de 1917 presentó dos de sus obras, de fuerte cromatismo ${ }^{20}$, en la exposición de Homenaje a los artistas franceses organizada por Dalmau en su galería. El 27 de agosto del mismo año visitó por primera vez a Joaquín TorresGarcía $^{21}$; éste publicaría en noviembre un artículo sobre Barradas en Montevideo en el que, aunque describe sus obras como "sinfonías en color" 22 , no menciona el vibracionismo. La presentación pública del movimiento tuvo lugar en diciembre de 1917, cuando Torres-García y Barradas expusieron juntos en las galerías Dalmau. Sin embargo, todo indica que en ese momento el concepto aún no había cuajado, pues Torres-García escribe que "Al voler batejar nostres assaigs de pintura [...] ens hem trobat amb gran dificultat per a expressar, per mitjà de la paraula, ço que ha trobat adequada expressió en la forma i el color", y propone el término, que no tendría continuidad, de "Plasticisme Biològic" 23 para referirse a la obra de ambos; de hecho las críticas de esta exposición no hacen alusión al vibracionismo. Según Antonio de Ignacios, hermano del pintor, la palabra "vibracionismo" sólo apareció públicamente por primera vez en la exposición que Barradas celebró en marzo de 1918 en las Galerías Laietanes, en la que una serie de obras se rotularon con este nombre ${ }^{24}$; en esta ocasión sí encontramos referencias al vibracionismo en las críticas publicadas ${ }^{25}$. Del hecho de que la adopción de la palabra "vibracionismo" fue una decisión plenamente cons-

${ }^{18}$ Carrá cita sus propias obras, junto a las de autores como Boccioni, Russolo o Severini; de este Severini cita específicamente el cuadro Le Pan-Pan (o La danse de Pan-pan au Monico, 1911), una posible influencia para Barradas.

${ }^{19}$ En una carta de Barradas a su íntimo amigo Julio J. Casal del 4 de abril de 1919 le cuenta su estancia en un hospital de Zaragoza en 1914, y como al despertar encontró "un albarán sobre mi cabeza con un marquito de hojalata que decía "Rafael Pérez Barradas" Nacionalidad Uruguay (Montevideo) Profesión Pintor Vibracionista." (reproducido en Pereda, 1989: 39). Considero muy improbable que en ese momento Barradas ya se llamara a sí mismo vibracionista, pero es posible que la palabra le rondara por la cabeza.

${ }^{20}$ García-Sedas, 1994: 47.

21 Torres-García, 1990: 136. Lubar, 1991: 26

${ }^{22}$ Torres-García, 1917b.

${ }^{23}$ Esta "Nota al públic de Torres-García" se reprodujo en el artículo no firmado "Les Exposicions. Exposició d'En Torres-García i exposició Barradas", La veu de Catalunya, 3-XII-1917.

${ }^{24}$ Ignacios, 1953: 62.

25 " "...] l'habilitat inquieta de Barradas, de ple en la més generosa recerca, féu vibrar l'esguard simplement tafaner dels espectadors d'un parpelleig sospitós". "A les Galeries Laietanes”, Trossos, nº 5, abril de 1918; “[...] ell es manifesta només amb vibracions, que són ses vibracions", T.R.S., "BARRADAS - Pintor vibracionista", Vell i nou, $\mathrm{n}^{\circ}$ 64, abril de 1918; pp. 134-135. 
ciente del pintor dan fe las palabras de su hermano, que vivía con él en Barcelona: "Recuerdo su alegría al hallar la justificación de esa palabra para su tendencia, concretando en síntesis su arte. [...] Decía: Sí, yo soy esto: "VIBRACIONISTA"26.

Sin embargo, existe una mención escrita al vibracionismo anterior a esta fecha en la portada del único número de Arc-Voltaic, publicada en febrero de 1918. La revista fue impulsada por el poeta Joan Salvat-Papasseit con la colaboración de Barradas y Torres-García; las frases "Plasticitat del vertic - Formes en emoció i evolució - Vibracionisme d'idees - Poemes en ondes hertzianes"27 han sido consideradas como una referencia a sus respectivos idearios estéticos. Por ello, podemos afirmar que aunque la plástica vibracionista se desarrolló a lo largo de 1917, el término sólo quedó fijado en algún momento entre la exposición de diciembre de 1917 y la publicación de Arc-voltaic, presentándose públicamente en la exposición de marzo de 1918. El vibracionismo siguió desarrollándose durante la estancia de Barradas en Madrid, donde llegó en agosto de 1918, como demuestran las críticas a sus exposiciones de $1919^{28}$ y $1920^{29}$; y se alargó posiblemente hasta principios de $1920^{30}$, aunque en realidad es imposible delimitar rígidamente los sucesivos "ismos" barradianos ${ }^{31}$.

En el vibracionismo barradiano resuenan algunos de los matices que, como hemos visto, había adquirido el concepto de vibración en el contexto de la vanguardia europea (especialmente el futurismo), aunque resulta prácticamente imposible documentar la relación de Barradas con la misma. Sabemos que el pintor llegó a Génova procedente de Uruguay en septiembre de 1913, y que antes de establecerse en Barcelona en mayo-junio de $1914^{32}$ estuvo en Milán, París y Suiza ${ }^{33}$. Su obra pictórica del momento (Estudio, MNAV, 1914) (fig. 1) prueba su conocimiento de las vanguardias artísticas, particularmente el futurismo y el cubismo; su periplo fue breve, pero coincidió con un momento especialmente estimulante en el ambiente artístico de Milán y París. Aunque con toda probabilidad no conoció de primera mano muchas de las referencias y autores que hemos citado, algunos de esos textos, especialmente los futuristas, sí estuvieron a su alcance, directa o indirectamente; igualmente, aunque no hubiera conocido toda la diversidad de la pintura del momento pudo acceder a muchas obras mediante reproducciones ${ }^{34}$. En cualquier caso, hay que señalar que entre su contacto con la vanguardia pictórica entre 1913-14 y la eclosión del vibracionismo en 1917 se produce una pausa; el material visual e intelectual que acumuló en su recorrido europeo no se concretaría en una propuesta artística autónoma y vanguardista hasta su segunda estancia en

${ }^{26}$ Ignacios, 1953: 62

27 Salvat-Papasseit, 1918.

${ }^{28}$ Su primera exposición individual en Madrid fue en abril de 1919 en la librería Mateu; la tercera sección de la misma se titulaba "vibracionismo", y en el catálogo algunas obras aparecían como vibracionistas. En su crítica de la exposición José Francés escribió que "Desde luego lo más interesante de su Exposición eran las notas vibracionistas [...] es en sus páginas vibracionistas dónde encontramos, por ahora, íntegro al artista.” (Francés, 1920: 118-119).

${ }^{29}$ En la exposición que presentó en el Ateneo de Madrid en marzo de 1920 aún se expusieron obras vibracionistas, algunas pintadas en Barcelona y otras en Madrid, y así lo reflejó la crítica: "Yo pienso que Barradas es el hombre superior de la escuela vibracionista", Correa-Calderón, 1920: 157.

${ }^{30}$ Aunque algunos autores consideran que cuando se presentó la exposición de 1919 ya había empezado a desarrollar su siguiente etapa (Carmona, 2012: 78), él aún se consideraba vibracionista; la carta a Casal de abril de 1919 termina así: "otra vez te hablaré de mi pintura. EL VIBRACIONISMO" (reproducido en Pereda, 1989: 102). La carta a TorresGarcía que citaré posteriormente en qué describe una experiencia vibracionista es de 28 de septiembre de 1919, y data la experiencia en cuestión unos 4 o 5 meses antes, es decir entre abril y mayo. En 1920 aún expuso obras vibracionistas.

${ }^{31}$ Carmona, 2012: 80. La tendencia que sigue al vibracionismo ha sido llamada "cubismo", pero el propio Barradas habló ya en noviembre de 1918 de "cubo-vibracionismo" (García-Sedas, 2001: 116); quizá lo más apropiado sea considerar que por un tiempo ambas tendencias se desarrollaron simultáneamente.

${ }^{32}$ Santos Torroella, 1993.

${ }_{33}$ Pereda, 1989: 28-29. Ignacios, 1953: 39.

${ }^{34}$ Para algunas hipótesis sobre qué pudo ver durante su periplo europeo, ver Pereda, 1989: 35.

Arch. esp. arte, LXXXVIII, 351, JULIO-SEPTIEMBRE 2015, 281-298

ISSN: 0004-0428, eISSN: 1988-8511, doi: 10.3989/aearte.2015.17 


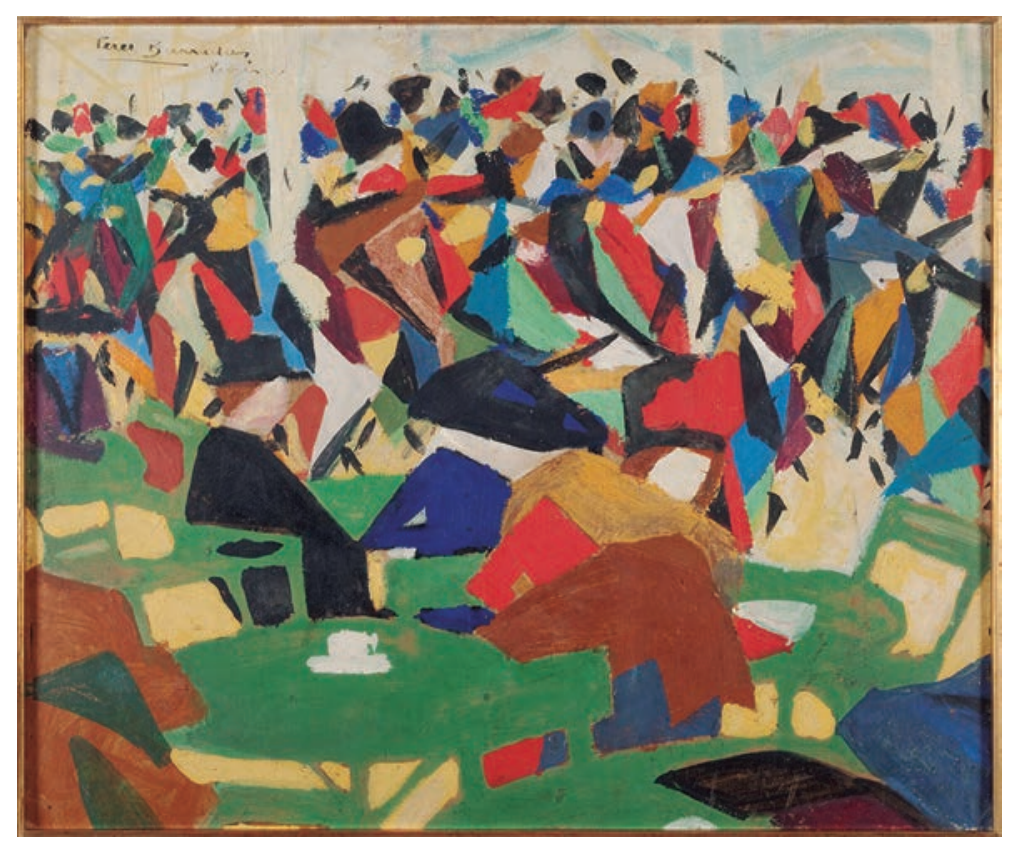

Fig. 1. Estudio (1914).

Óleo s. cartón, 46 x $54 \mathrm{~cm}$. MNAV, Montevideo.

Barcelona ${ }^{35}$. Sin embargo, y aunque durante su periodo en Zaragoza su pintura pertenece a una estética entre simbolista y naturalista, no olvidó lo que había descubierto; así, el crítico Ángel Abella escribiría de Barradas en 1915 que "En todo ve el color: en el sonido de las palabras, en la armonía de una sonata, en el ritmo del baile, en el ruido de un tranvía al deslizarse sobre sus rails. El cromatismo le enajena." ${ }^{36}$, lo que demuestra que su interés por la multisensorialidad estaba vivo durante estos años, aunque fuera en estado latente.

En Barcelona Barradas pudo retomar el contacto con la vanguardia principalmente gracias a las exposiciones programadas en las Galerías Dalmau; de ellas, quizá las más relevantes en relación al tema que nos ocupa fueron las de Serge Charchounne en 1916 y 1917 y la de Albert Gleizes en 1916. La primera muestra de Charchounne presentó ejemplos de lo que él mismo calificó como "cubismo ornamental", que parte del concepto lineal y rítmico del arabesco para crear obras abstractas. La segunda exposición se tituló Art ornamental. Films, y en ella se mostraron una serie de óleos abstractos pensados como secuencias de una película de animación. Las críticas reconocieron perfectamente esta dimensión sinestética y temporal de las obras, como escribieron Joan Sacs ${ }^{37}$ o Solé de Sojo: "Un sentiment pot ésser expressat per la vida material del color; una harmonía musical pot ésser traduïda per una línia dotada de movilitat" ${ }^{\prime 38}$. Gleizes presentó en diciembre de 1916 su serie de las Bailarinas españolas, en la que trabajó la relación entre danza, color y música, una vertiente de la investigación sinestética que había interesado a numerosos artistas, como Sonia Delaunay. Precisamente el proyecto

${ }^{35}$ Aunque ya durante la primera había dejado algunas pistas de su interés vanguardista, como el dibujo La Bailarina (dibujo futurista) (col. part.); Santos Torroella, 1993. Según parece, en 1916 no pintó nada (Pereda, 1989: 62).

${ }^{36}$ Abella, 1915: 4. El conjunto del artículo sugiere que este comentario, como alguna anécdota que incluye, se derivaría de una conversación con el propio Barradas.

${ }^{37}$ Sacs, 1917: 8

${ }^{38}$ Solé de Sojo, 1917: 1. El texto sigue: "Vegi’s el seu film "Guitarre" [...]. Les línies de la guitarra, les cordes, el vibrar d'aquestes, les evocacions que la guitarra desperta, [...]. Hi ha moments en què no sabem si ens trobem davant una composició d'art plàstic o una composició musical." 
de Dalmau de realizar en su galería una exposición sobre el Simultaneísmo con obras de los Delaunay y otros autores internacionales, que habría estado acompañada de conciertos, conferencias, películas y danza, y que habría convertido las Galerías en un entorno simultáneo total ${ }^{39}$, debió hacer que ya en 1916 las relaciones entre las artes fueran un tema de conversación en la galería, un espacio muy frecuentado por Barradas. Cabe señalar también la presencia en Barcelona, entre julio de 1916 y marzo de 1918, de un artista tan interesado por las relaciones interartísticas como Francis Picabia ${ }^{40}$.

El de vibración es un término que apareció con cierta frecuencia en el contexto de la vanguardia catalana, por ejemplo, en diversos textos del crítico y poeta Josep $\mathrm{M}^{\mathrm{a}} \mathrm{Junoy}$. En el primer número de su revista Troços ${ }^{41}$ se publicó la prosa "El Jean Cocteau d'Albert Gleizes", que empieza con la frase "Cerebral concreció d'una verticalitat en cadencia complexa que vibra màgica amb suaus inclinacions de palma esvelta" ${ }^{42}$. En el mismo número apareció el caligrama Films. Sergi Charchoun$n e$, en el que Junoy alterna una partitura del músico futurista Balilla Pratella con su propio texto, el segundo párrafo del cual reza lo siguiente: "germes abstractes punts i ratlles inicials simulacres florades simetries en lepidóptera vibració"; y más tarde escribiría que en los poemas de Solé de Sojo encontramos la "vibratilitat kaleidoscòpica de Gino Severini" ${ }^{\text {". }}$. El 22 de enero de 1917 Junoy publicó en El poble català un texto sobre la muerte de Boccioni cuya última línea reza "Boccioni vibra ara en pau". La relativamente frecuente aparición del concepto de vibración en ambas publicaciones resulta especialmente significativa sobre todo si pensamos que en 1917 Junoy y Solé de Sojo defendieron la cercanía entre cubismo y futurismo y la necesidad de su convivencia ${ }^{44}$, hasta el punto de que incluso Sunyer, comparado con el "vibrante" Boccioni y con Severini, fue considerado un pintor próximo al futurismo, y su trabajo fue descrito en unos términos que más parecerían corresponderse con la obra vibracionista de Barradas ${ }^{45}$.

El ya citado encuentro entre Torres-García y Barradas fue sin duda determinante para ambos, y punto de partida de una entrañable relación personal y artística. 1917 fue un año clave para Torres-García, puesto que a lo largo del mismo se confirmó la evolución de su obra desde el Noucentisme hasta las posiciones de vanguardia ${ }^{46}$; en este proceso destaca su colaboración con Salvat-Papasseit en la revista editada por éste Un enemic del poble, en la que también participaría Barradas. Su texto para el número de agosto consta de tres fragmentos titulados "Una vibració", "Un crit", "Un riure intel-ligent" ${ }^{47}$ y empieza así: "Una vibració, un instant, un acord d'idees i de mots, de sons, a banda i banda del no-res". La relación entre Torres, Barradas y Salvat debió ser especialmente intensa entre el otoño de 1917 y el invierno de 1918; precisamente por medio de Salvat pudo llegar al grupo otra referencia importante a las vibraciones ${ }^{48}$. Como vemos, el concepto

\footnotetext{
${ }^{39}$ Rousseau, 2005: 332.

${ }^{40}$ Picabia había tratado la representación plástica de la danza en obras como Udnie (1913), y las analogías entre música y pintura en la acuarela abstracta La musique est comme la pinture, pintada entre 1913-16.

${ }^{41}$ El nombre de esta revista aparece reproducido en al menos dos obras de Barradas, Affiche (1917, MNAV) y Acuarela (1917, MNAV).

${ }^{42}$ Trossos, $1916, \mathrm{n}^{\circ} 0$

${ }^{43}$ Trossos, 1917, $\mathrm{n}^{\circ} 3$

44 Vallcorba, 1984: LXVI y sigs.

45 "Ecos", El poble català, 18-II-1917; aunque no está firmado, el autor es Solé de Sojo. En el "eco" anterior él mismo se refiere a "les vibracions més exaltades dels sentits" que caracterizaron el cambio de siglo.

${ }^{46}$ Lubar, 1991.

47 Torres-García, 1917a: 1.

${ }^{48}$ Puesto que la serie de haikús que Salvat incluyó en su libro L'irradiador del port i les gavines (1921) se tituló precisamente "Vibracions", se ha señalado (Mas López, 2004: 189-234) que Salvat, Torres y Barradas pudieron estar influidos en su uso del concepto de vibración por el texto del francés P.-L. Couchod "Les épigrammes lyriques du Japon" (reditado en 1916), en el que escribe de los haikús: "Ils sont semblables à une vibration qu'acune autre ne limite et qui s'élargit d'elle même presque indéfiniment" (citado en Mas López, 2004: 208-209).
}

Arch. esp. arte, LXXXVIII, 351, JULIO-SEPTIEMBRE 2015, 281-298

ISSN: 0004-0428, eISSN: 1988-8511, doi: 10.3989/aearte.2015.17 
de vibración formaba parte del vocabulario artístico utilizado por el sector artístico más vanguardista e informado de Barcelona, precisamente en el momento en qué Barradas, que participaba plenamente de este contexto, decidió adoptarlo como término definidor de su estética.

\section{"Sólo lo que se mueve existe"}

La definición más citada sobre el vibracionismo es la que Torres-García incluyó en el capítulo dedicado a su amigo en Universalismo constructivo (1944):

"El vibracionismo es, pues, cierto MOVIMIENTO que se determina fatalmente por el paso de una sensación de color a otra correspondiente, siendo cada uno de estos acordes diversas notas de armonía, distintas, fundidas entre sí por acordes más sordos, en gradación cada vez más opaca" ${ }^{49}$.

En este texto queda claro que la definición no es sólo cosa suya, si no que surgió de un diálogo con Barradas: "Un día quise concretar el arte de Barradas, y nos pusimos a definirlo bien; él hablaba y yo le contestaba, preguntando al mismo tiempo, y a veces deteniéndonos a considerar bien las $\operatorname{cosas}{ }^{50}$. En realidad, y este hecho ha sido habitualmente ignorado, parte del texto se había publicado mucho antes; el 7 de abril de 1919 apareció en el periódico El Heraldo de Madrid una crítica de la exposición que el pintor uruguayo celebraba en la Librería Mateu titulada "Exposición del vibracionista Barradas" y firmada por José Blanco Coris, en la que leemos lo siguiente:

"El vibracionismo es, según los precursores del movimiento modernista, cierto movimiento que se determina "fatalmente" en el artista por el paso de una sensación de color a otra correspondiente siendo cada uno de estos acordes adversas notas de armonía distintas, fundidas entre sí por acordes más sordos en gradación cada vez más "opaca". La forma del vibracionismo es la geométrica, y estas formas se traducen arbitrariamente para ser traducidas por el espectador" ${ }^{\prime \prime}$.

El hecho de que ambos textos sean prácticamente idénticos sugiere que en 1919 la definición del vibracionismo estaba perfectamente fijada; ello probaría la participación del propio Barradas en la redacción de la misma (aunque fuera mediante el diálogo con Torres-García), y también que fue él mismo quién la difundió, quizá imprimiéndola en el catálogo de dicha exposición. El texto publicado por Torres-García, más completo, adquiere así más valor, puesto que en él oímos ahora con más fuerza la voz del mismo Barradas.

La noción de vibracionismo que se propone en dicho texto evoca algunas ideas que hemos citado en el primer apartado. Así, la obra surge cuando el artista fija la atención en algún objeto, sin que ni él mismo pueda decir porqué, "pues el artista también lo ignora" 52 . A partir de la emoción generada por este primer objeto, busca la "asociación de emociones o sensaciones correspondientes a esa primera, centro de una misma calidad o saber plástico. E inmediatamente, a esta primera asociación seguirán otras, como dentro de una escala propia de valores, buscando del mismo modo sus correspondientes y enlazándose todas en gradación"s33. Las palabras 'asociación' y 'correspondencias' forman parte del mismo vocabulario plástico al que pertenece el concepto de la vibración, y aluden precisamente a cómo lo visible y lo invisible (objetos y emociones, en este caso) se re-

\footnotetext{
49 Torres-García, 1984: 477; las cursivas y mayúsculas están en el original.

${ }^{50}$ Torres-García, 1984: 477. El texto se publicó en 1944, aunque se redactó en 1936; la conversación en cuestión debió tener lugar entre 1917 y 1918, cuando ambos vivían en Barcelona.

${ }^{51}$ Blanco Coris, 1919; el autor era, además de crítico, un pintor conservador del cual no se conoce ninguna relación específica con los círculos de vanguardia.

52 Torres-García, 1984: 477.

53 Torres-García, 1984: 477.
} 
lacionan entre sí. La noción de irradiación de emociones o sensaciones desde un centro nos remite a la imagen de las ondas producidas por la caída de una piedra en un estanque, que por analogía está en el origen mismo de las teorías sobre la vibración de la luz ${ }^{54}$.

El vibracionismo se concreta pues como el "MOVIMIENTO (...) de una sensación de color a otra correspondiente". La incorporación del movimiento, única palabra en mayúsculas, a la pintura fue uno de los objetivos de diversos movimientos de vanguardia; aquí se habla concretamente del movimiento de un color a otro, lo que nos remite a un objeto que jugó un papel importante, no del todo reconocido, en las primeras vanguardias: los discos de color en movimiento de rotación ${ }^{55}$. El efecto de mezcla óptica y modificación cromática continua producido por estos discos ejerció una influencia y una sugestión visual determinante en obras concretas de pintores como Delaunay ${ }^{56}$, Kupka o los sincromistas Russell y Macdonald-Wright, todos los cuales escribieron sobre ellos y el efecto dinámico que producían; a Kupka, en particular, le fascinaba como consiguen "donner l'impression du mouvement en employant des moyens en eux-mêmes immobiles!" 57 . Y ya hemos visto que para Delaunay la cuestión de las vibraciones de los colores yuxtapuestos era importante en función del "movimiento" que imprimían a la pintura. La cuestión del movimiento del color implica un factor de duración temporal y favorece, así mismo, una analogía entre la música y la pintura que algunos autores recogieron. Según el texto de Carrá que hemos citado, la propia incorporación de elementos multisensoriales ya es una alusión al movimiento; y quizá sea de interés recordar que Torres-García le escribió a Barradas en noviembre de 1919 que "creo que sólo lo que se mueve existe" ${ }^{58}$. La referencia a la gradación, recurrente a lo largo de todo el párrafo dedicado al vibracionismo, para referirse al paso de unas sensaciones o colores a otros reforzaría la referencia a los discos, que Barradas pudo conocer aunque fuera indirectamente a través de las obras de los artistas citados (especialmente Delaunay, con quién el vibracionismo tiene la mayor afinidad) ${ }^{59}$.

El texto adopta enseguida un vocabulario musical y describe las sensaciones de color como "acordes", "diversas notas de armonía" ${ }^{60}$ distintas y a la vez fundidas entre sí; el uso de este vocabulario para referirse a la pintura fue un recurso muy utilizado por artistas y críticos desde el Simbolismo, y como hemos visto la comparación color-sonido está en el corazón mismo de la teoría de las vibraciones. De cómo Torres-García entendía que el factor multisensorial era fundamental para el vibracionismo dan fe otros fragmentos del mismo texto; así escribe que los gouaches de Barradas que vio en 1917 eran "traducción en plástica de la realidad captada con toda integridad, sin que se escapase olor ni sabor, ruido o movimiento, expresión o calidad, síntesis, pintura" ${ }^{61}$. Y al final, añade que Barradas en su obra no describía, sino que fundía "en el elemento plástico, en color, en forma y en calidad, en ritmo, en geometría, o en arabesco ${ }^{62}[\ldots]$ Su obra, pues, es una orquesta plástica" ${ }^{\prime 3}$. Por lo que se refiere al papel de la música en la obra de Barradas, es necesario recordar que su hermana Carmen era una pianista y compositora que participó del mismo contexto

54 Roque, 2002: 180. Muchos artistas finiseculares crearon obras en las que las ondas de colores evocan también la noción de vibración, como por ejemplo Munch, Mondrian o los simbolistas holandeses Toorop y Thorn Prikker.

${ }^{55}$ Como los discos de Newton o los discos de Maxwell, a los que diversos autores (entre ellos Chevreul) dedicaron textos muy conocidos entre los artistas. Roque, 2009: 408 y sigs.

56 Roque, 2002: 184.

57 Kupka, 1989: 198.

58 García-Sedas, 1994: 185.

59 Santos Torroella, 1993: 62.

60 Torres-García, 1984: 477.

61 Torres-García, 1984: 475.

62 Sobre las relaciones entre movimiento, música y color evocadas por la noción de "arabesco" ver Rousseau, 2003.

63 Torres-García, 1984: 479.

Arch. esp. arte, LXXXVIII, 351, JULIO-SEPTIEMBRE 2015, 281-298

ISSN: 0004-0428, eISSN: 1988-8511, doi: 10.3989/aearte.2015.17 
artístico que él y jugó un papel significativo en algunos de sus trabajos ${ }^{64}$, llegando ambos incluso a trabajar juntos en la creación de obras, partiendo cada uno de la música o la pintura que el otro elaboraba ${ }^{65}$.

La reiteración de la metáfora musical para expresar el intento barradiano de recoger en la obra la simultaneidad sensorial remarca el hecho de que es precisamente el concepto de vibración el que permite establecer un nexo entre las experiencias. Esto queda muy claro, desde una perspectiva más subjetiva, en una carta que Barradas escribió a Torres-García en septiembre de 1919: “[...] hace cosa de cuatro o cinco meses, un día, estando VIENDO en un café, pasó un batallón, es decir, unos sonidos de trompas y tambores y unas campanas de tranvías. Simultáneamente sonaba un piano en el café, pero que quedaba fuera del café. VIBRABAN todas las cosas, que, en realidad no lo son. YO VIBRABA de tal manera que CREABA las COSAS, -y como digo- me pasó lo que ahora: me hubiera pegado un tiro en la cabeza, precisamente en la CABEZA" ${ }^{" 66}$.

No se trata sólo, pues, de que lo que el pintor ve y oye vibre en él al unísono, es decir, que él como artista-espectador lo capte simultáneamente en toda su riqueza sensorial; sino que además es él mismo quién vibra, y al hacerlo, crea la experiencia que está viviendo. Así, aunque no se encuentran en el Barradas de esta época referencias claras a un interés por lo espiritual, no debe extrañarnos que Torres-García cite las siguientes palabras de su amigo: "yo creo, Torres, que nosotros llegamos a eso que los teósofos llaman la mente superior, o cosa así, porque hay momentos en que uno ya no es uno mismo"67; lo cual no implica ninguna afiliación de Barradas con la teosofía, sino simplemente la constatación por su parte de la potencia irracional del acto creativo vivido con total intensidad, a lo que aludiría la propia imagen del disparo, precisamente, en la cabeza. Este poder creativo no es exclusivo del artista, sino que también pertenece al espectador de la obra vibracionista; volviendo al texto sobre el vibracionismo, lo que el pintor produce plásticamente, aun creando formas "GEOMETRIZADAS", es decir, no imitativas del mundo visible, lo "completa" el espectador: "[...] así logra el artista otro modo de vibración, algo viviente QUE NO DARÍAN LOS OBJETOS REPRESENTADOS NORMALMENTE O COMPLETOS" ${ }^{\prime 8}$. La vibración reverbera entre creador, obra y espectador, creando una conexión entre ellos, como hemos visto en el primer apartado.

$\mathrm{Al}$ empezar hemos indicado que las fuentes plásticas del vibracionismo las proporcionaron el futurismo, el simultaneísmo y el cubismo, movimientos que Barradas no exploró a fondo, sino que obtuvo de ellos una serie de referencias visuales a partir de las cuales desarrolló su propia síntesis ${ }^{69}$. En este contexto, la adopción del concepto de vibracionismo le permitió poner en juego diversos elementos que estaban presentes, en distinto grado, en la cultura vanguardista contemporánea. Por un lado, el concepto de vibración aludía a la afinidad entre colores y sonidos (musicales o no), lo que le permitió incorporar la multisensorialidad a sus obras; por otra parte, la vibración de los colores también podía aludir al anhelo de introducir el dinamismo en la pintura. Finalmente el concepto de vibración contiene un factor emocional que sugiere la vinculación irracional entre el artista y el mundo que le rodea; Salvat-Papasseit ya puso de relieve que el vibracionismo "en realidad es vibración, es decir sensación" "70. Los aspectos sinestético, dinámico y emocional del concepto de la vibración son tres facetas del vibracionismo como intento de transcribir en la tela, de forma no naturalista, la simultaneidad de sensaciones subjetivas tal y como son percibidas por el artista.

\footnotetext{
${ }^{64}$ Piñero, 2009.

${ }^{65}$ Pereda, 1989: 74.

${ }^{66}$ García-Sedas, 1994: 136.

${ }^{67}$ Torres-García, 1984: 479.

${ }^{68}$ Torres-García, 1984: 478.

${ }^{69}$ Brihuega, 1993: 20-22.

${ }^{70}$ Roig, 1992: 84.
} 


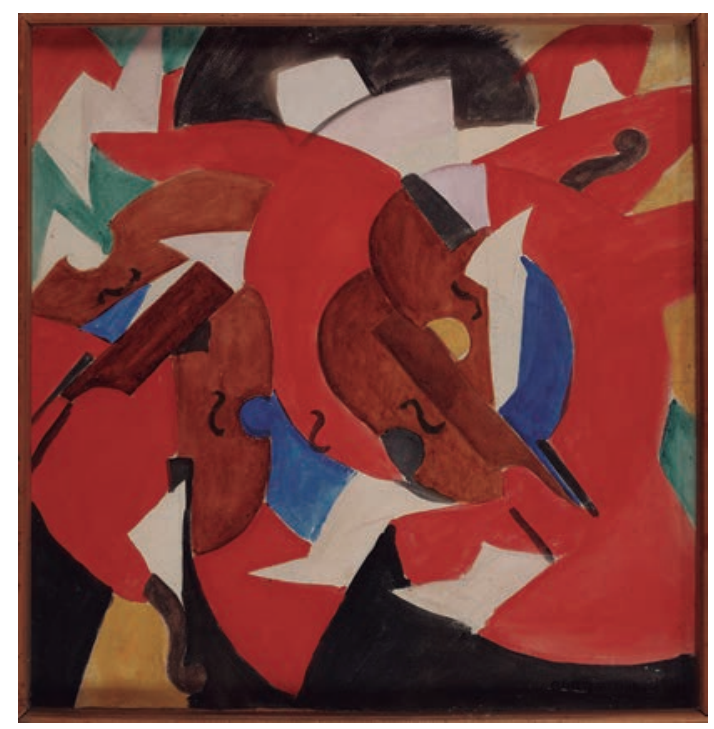

Fig. 2. Violinista (1917). Acuarela s. cartón, 52 x $48 \mathrm{~cm}$. MNAV, Montevideo.

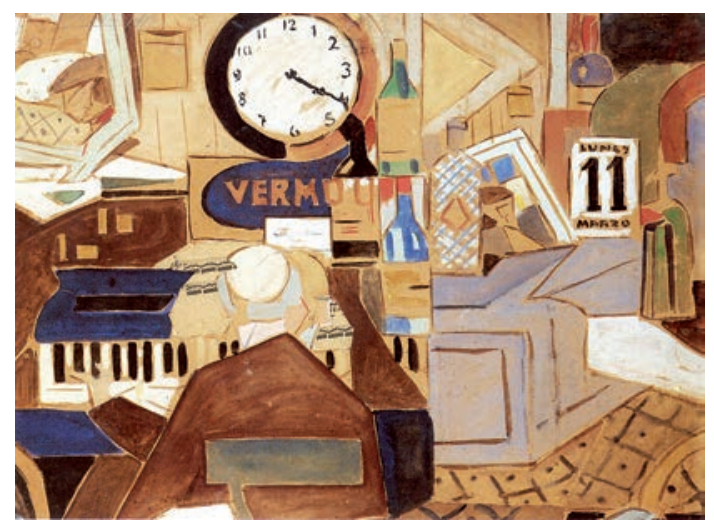

Fig. 3. Collage vibracionista (1918). Técnica mixta, 45 x $63 \mathrm{~cm}$. Colección particular, Buenos Aires.

\section{"De su paleta nacen rayos de luz"}

Lo que une al conjunto de obras vibracionistas de Barradas es pues precisamente la voluntad de captar lo que ha sido definido como "el continu trànsit entre subjecte i objecte, entre consciència i món circumdant" ". Es lo que su hermano Antonio describió como "esa emotividad nerviosa del espíritu en una entremezclada simultaneidad de sensaciones nuevas" ", que podría equipararse a la "vibración universal" de la que hablaba Marinetti ${ }^{73}$. Para ello Barradas se servirá de una plástica figurativa pero no naturalista, de un cromatismo intenso y saturado, de la fragmentación de los elementos en planos angulosos o fluidos, introduciendo en ocasiones un factor dinámico, e incorporando letras, números, y puntualmente collage. Puesto que la obra vibracionista nace de una experiencia total y completamente subjetiva del artista respecto a la realidad que le rodea, no puede ser plásticamente homogénea; y aunque la diversidad formal está presente desde el principio, con el tiempo (sobre todo a partir de 1919) habrá otros cambios: el colorismo y el dinamismo se atenuarán y el interés por la estructura y composición de las obras se acentúa.

La vibración como metáfora de la conexión perceptiva entre el artista y su entorno impregna pues la obra vibracionista; una de sus vertientes es la dimensión específicamente plurisensorial implícita en el concepto de vibración, un aspecto fundamental para comprender la apropiación barradiana del término, como prueban por ejemplo las obras vibracionistas relacionadas con la música. Entre ellas podemos destacar la acuarela Violinista (1917, MNAV) (fig. 2), en la que Barradas va más allá del violín como elemento iconográfico ${ }^{74}$ para evocar el efecto dinámico del movimiento del instrumento en el espacio. Un instrumento que aparece más frecuentemente en sus obras es el piano, que aparece en retratos familiares (Carmen, 1920, MNAV o Retrato de Antonio, 1920 c.,

\footnotetext{
71 Carmona, 1993: 113.

72 Ignacios, 1953: 62.

73 Marinetti, 1996: 100.

${ }^{74}$ El pintor futurista Giacomo Balla pintó La mano del violinista o Ritmo del violinista (1912, col. part.).
}

Arch. esp. arte, LXXXVIII, 351, JULIO-SEPTIEMBRE 2015, 281-298

ISSN: 0004-0428, eISSN: 1988-8511, doi: 10.3989/aearte.2015.17 


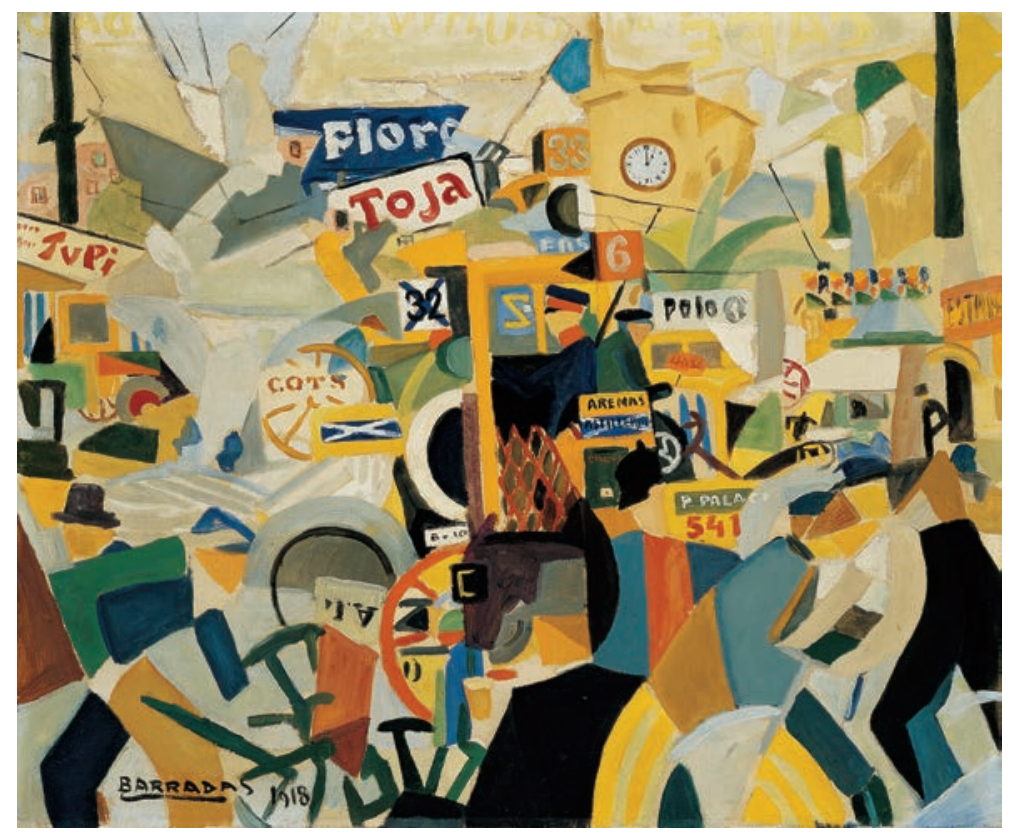

Fig. 4. Calle de Barcelona (1918). Óleo s. lienzo, 50,7 x $60,5 \mathrm{~cm}$. C.A.C. SGL Carbón, S.A.- Museo Patio Herreriano, Valladolid.

Col. Telefónica) o en obras como el Collage vibracionista (1918, col. part.) (fig. 3), en el que la música es citada directamente mediante unos fragmentos de una partitura pegados en la tela. Una última obra muy sugerente es el Retrato de familia (1920 c., localización desconocida), de la última etapa vibracionista; es un tema que Barradas trató en múltiples ocasiones, pero que aquí destaca por su abstracción y por la referencia a la música que supone la inclusión de las palabras "BachFugas". Bach, cuya obra fue uno de los grandes redescubrimientos musicales de principios de siglo, fue un tema clave y recurrente para el arte de vanguardia ${ }^{75}$, y el concepto de "fuga" en sí mismo sirvió como referencia e incluso título para obras de muchos pintores. Cabe recordar también que Salvat incluyó en sus Poemes en ondes hertzianes (1919, con textos escritos desde 1917) un texto titulado El record d'una 'Fuga' de Bach'.

En otras piezas Barradas intenta incorporar el sonido urbano en general, incluido el ruido. En el ya citado único número de Arc-Voltaic se publicó el Dibuix vibracionista conocido como "Bonanitingui" (1917, IVAM), una escena urbana y nocturna de la que se intentan reproducir diversos efectos lumínicos (luz de la farola) y sonoros (palmada, ruido de llaves, saludo del sereno), mientras un reloj resalta la dimensión temporal ${ }^{77}$. La misma voluntad de incorporar a la obra visual los sonidos de la calle se encuentra, creo, en un buen número de escenas urbanas que Barradas pintó entre 1917 y 1919, como Quiosco de Canaletas (1918, MALBA), Calle de Barcelona (1918, C.A.C. - Museo Patio Herreriano) (fig. 4), Estudio (c. 1918, MNAV) o Acuarela "Café Gran Hotel" (1918, MNAV), por señalar algunas; en estas obras no hay onomatopeyas, pero si muchas palabras que corresponden a carteles, anuncios y otros signos verbales habituales en el paisaje urbano, y cuya presencia no apunta sólo a su dimensión gráfica sino también a su vertiente sonora, puesto que toda palabra existe para ser dicha.

\footnotetext{
${ }^{75}$ Maur, 2004.

${ }^{76}$ Salvat-Papasseit, 1919.

${ }_{77}$ Sobre la abundancia de relojes en la iconografía de Torres-García y Barradas de la época, ver Bonet 1993.
} 

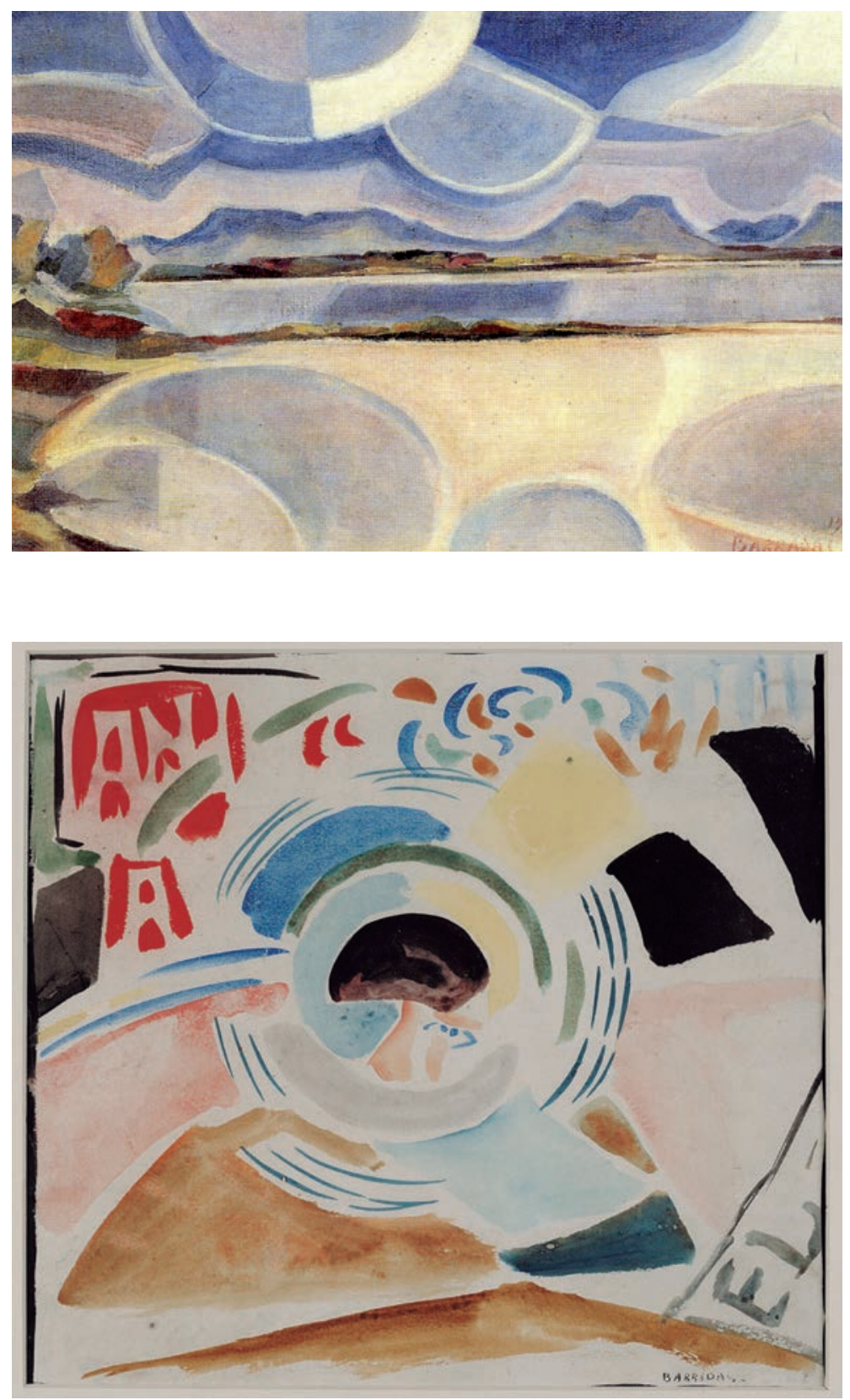

Fig. 5. Paisaje vibracionista (1918). Óleo s. tela, $27 \times 41,5 \mathrm{~cm}$. Colección privada.
Fig. 6. La taza de café (1917 c.). Acuarela s. papel, $25 \times 26 \mathrm{~cm}$. MNAV, Montevideo.

Otro grupo de obras opta por explorar la relación entre luz, color y movimiento; serían las que más elementos formales tienen en común con la pintura de los ya citados Delaunay, Kupka o los sincromistas. En estas obras, como el collage Affiche (1917, MNAV), Paisaje vibracionista (1918, colección privada) (fig. 5), Composición vibracionista (1917, MNBA) o La taza de café (1917 c., MNAV) (fig. 6) los círculos concéntricos pueden evocar formas cósmicas (sol, planetas, estrellas), así como los efectos físicos de la luz (halos creados por la luz artificial), las ondas vibratorias

Arch. esp. arte, LXXXVIII, 351, JULIO-SEPTIEMBRE 2015, 281-298

ISSN: 0004-0428, eISSN: 1988-8511, doi: 10.3989/aearte.2015.17 


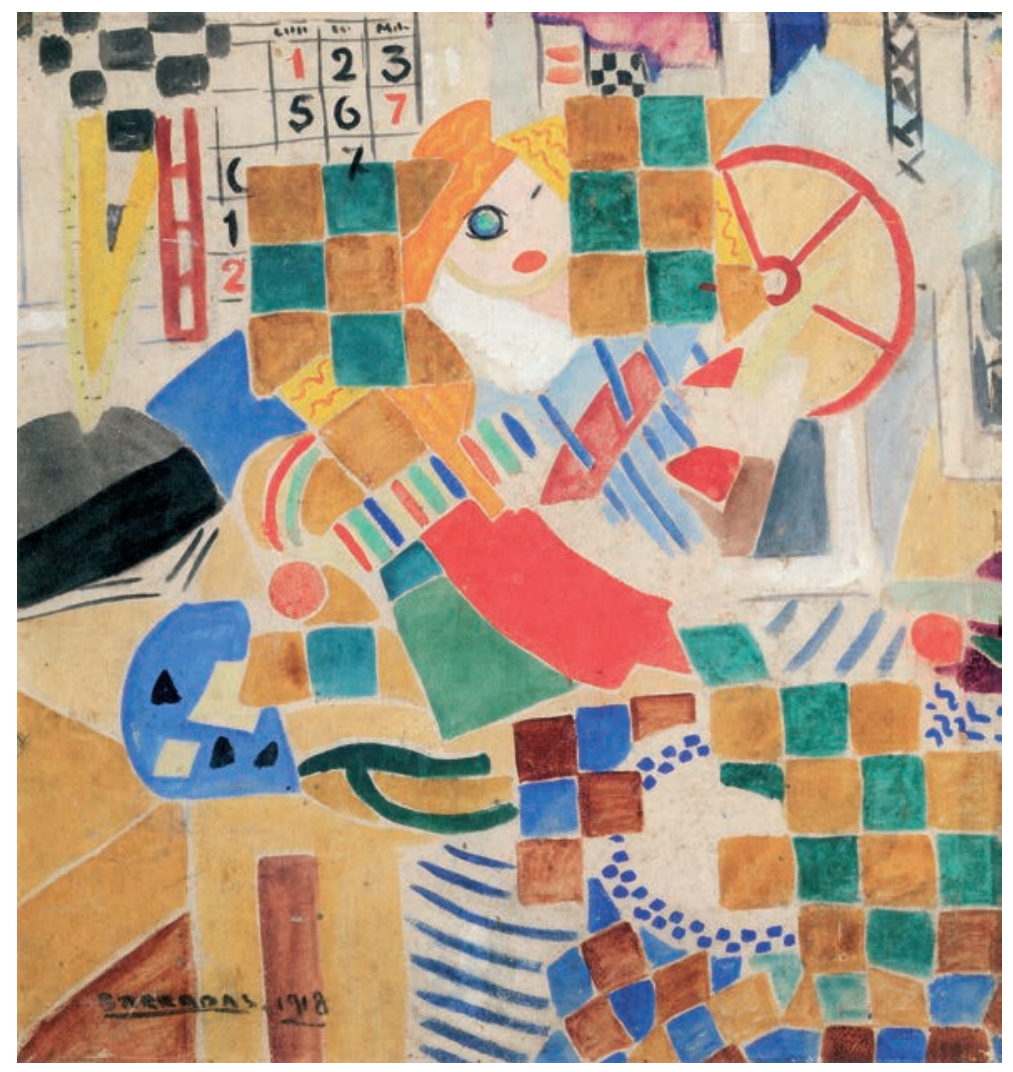

Fig. 7. Barcelona (1918).

Gouache s. papel,

48,5 x 44,5 cm. Museu

d'Història de L'Hospitalet.

acuáticas (que como hemos visto fueron el modelo para la teoría vibratoria de la luz) o los discos de colores a los que ya hemos aludido, a los que la forma circular cita directamente; en sus colegas europeos el círculo está directamente relacionado con la cuestión del movimiento de los colores en la superficie del cuadro ${ }^{78}$. Como nos recordaba la cita de Delaunay, los colores disonantes, vecinos en el círculo cromático, vibran más deprisa cuando son yuxtapuestos que los complementarios; así, la gama en azules de Paisaje vibracionista o Puerto, por ejemplo, generaría una cierta sensación de velocidad en la superficie pictórica, mientras que los contrastes más contundentes, como en La taza de café, serían más lentos. Con todo, no consta que Barradas conociera a fondo los tratados científicos sobre el color que sí manejaron Delaunay o Kupka, y por lo tanto su uso de los colores debe considerarse intuitivo; su paleta es, además, más apagada que la de los artistas a los que hemos hecho referencia. En la definición de vibracionismo antes citada se hace expresa referencia a que los colores se funden en "acordes más sordos" y "gradación más opaca"79.

En cualquier caso, el uso de formas circulares es un rasgo muy característico del vibracionismo barradiano; a menudo los círculos no se refieren a ningún elemento figurativo concreto, como en Quiosco de Canaletas, Jugadores de naipes (1917, col. part.) o el dibujo Escena de calle (1918 c.,

\footnotetext{
${ }^{78}$ Rousseau, 1999: 171.

79 Torres-García, 1984: 477.
} 


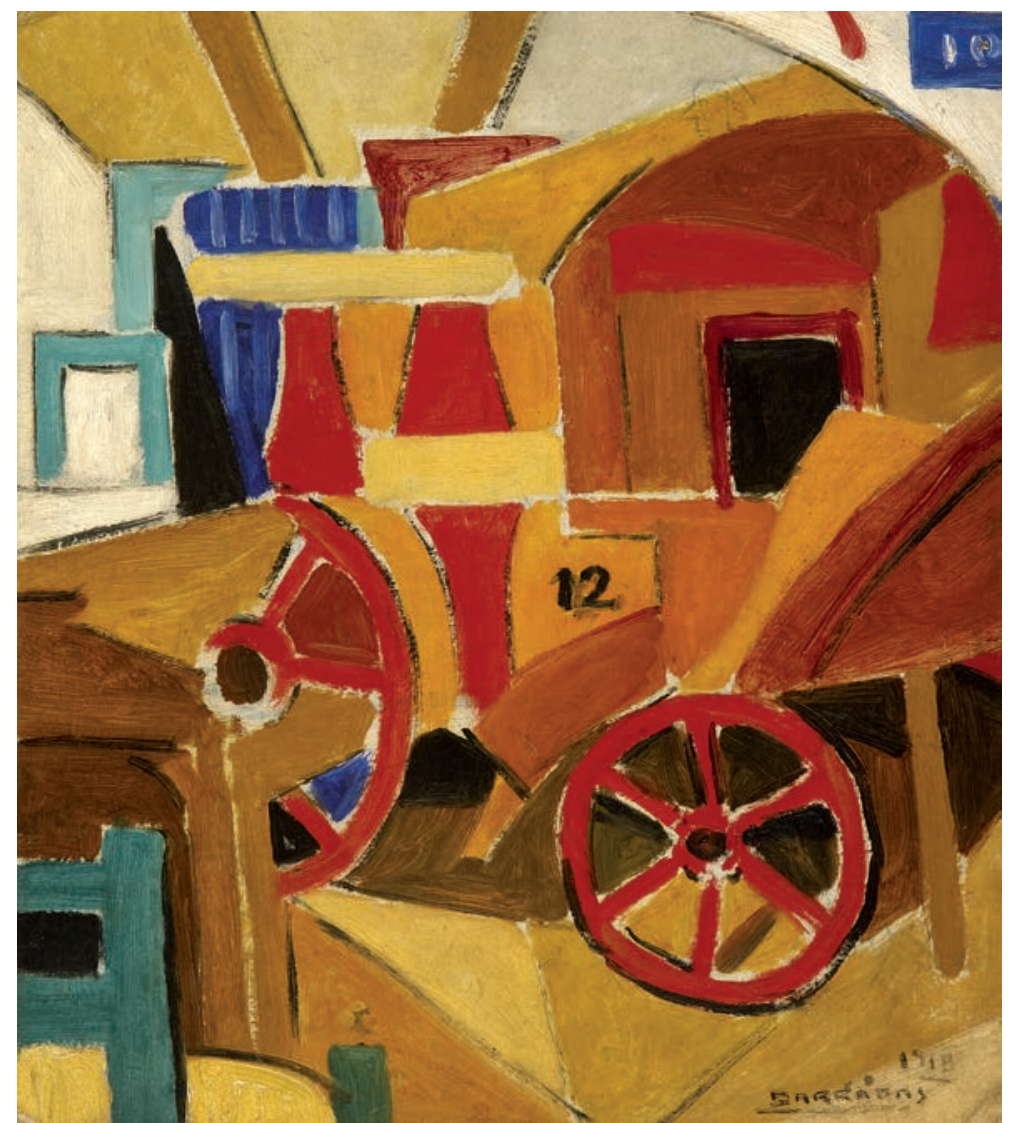

Fig. 8. La vieja carroza (1918). Óleo s. cartón, 47,3 x 41,5 cm. MALBA Fundación Constantini, Buenos Aires.

col. Jorge Mara). Sin embargo, en un grupo numeroso de obras vibracionistas aparece una forma circular específica, la rueda de carro, de color marrón, verde, y sobre todo de un rojo fuerte, como en Acuarela "Café Gran Hotel", Composición (1917, MNAV), Cadete (1918, MNAV), Barcelona (1918, Museu de l'Hospitalet) (fig. 7), El circo más lindo del mundo (1918, MALBA), La vieja carroza (1918, MALBA) (fig. 8) o Todo a 0,65 (1919, MNAV). Este círculo radial rojo, que encontramos coetáneamente en Torres-García (Composición vibracionista, 1918, col. part.) y un poco más tarde en Miró (La Masia, 1921, National Gallery of Art), ha sido tradicionalmente identificado con un símbolo solar ${ }^{80}$; entre el sol y el ojo, a su vez, se ha establecido a menudo una identidad física, que la teoría de las vibraciones con la que hemos empezado habría contribuido a explicar ${ }^{81}$. Quizá por ello Salvat-Papasseit escribió que de la paleta de Barradas "nacen rayos de luz, igual que si tuviera en la mano el arco iris" ${ }^{\prime 2}$, reconociendo así poéticamente la cualidad a la vez radiante y vibrante, en todos los sentidos, de su obra.

\footnotetext{
${ }^{80}$ Balsach, 2007: 41

${ }^{81}$ Rousseau, 2003.

${ }^{82}$ Roig, 1992: 84.
}

Arch. esp. arte, LXXXVIII, 351, JULIO-SEPTIEMBRE 2015, 281-298

ISSN: 0004-0428, eISSN: 1988-8511, doi: 10.3989/aearte.2015.17 


\section{BIBLIOGRAFÍA}

Abella, Ángel (1915): “Pérez Barradas". En: Paraninfo, no 47, pp. 3-4.

Balsach, M. Josep (2007): Joan Miró. Cosmogonies d’un món originari (1918-1939). Barcelona: Galaxia Gutemberg.

Blanc, Charles (1876): Grammaire des arts du dessin. París: Librairie Renouard.

Blanco Coris, J. (1919): “Exposición del vibracionista Barradas”, El Heraldo de Madrid, 7-IV.

Bonet, Juan Manuel (2003): “Alrededor del reloj: Joaquín Torres-García, Rafael Barradas, Joan Salvat-Papasseit”. En: Joaquín Torres -García, 1874-1949. Barcelona: AUSA y ICUB, pp. 88-108.

Brihuega, Jaime (1993): "Saturn al sifó. Barradas i l'avantguarda espanyola”. En: Brihuega, Jaime / Lomba, Concha (dirs.), Barradas. Exposició antològica, 1890-1929, L'Hospitalet de Llobregat: Centre Cultural Tecla Sala, pp. 13-45.

Carmona, Eugenio (1993): “Rafael Barradas i l'«art nou» a Espanya. 1917-1925”. En: Brihuega, Jaime / Lomba, Concha (dirs.), Barradas. Exposició antològica, 1890-1929. L'Hospitalet de Llobregat: Centre Cultural Tecla Sala, pp. 107-139.

Carmona, Eugenio (2012): “Bodegón. 1919”. En: DD.AA, Colección cubista de Telefónica, Madrid, Fundación Telefónica, pp. 76-80.

Correa-Calderón, Evaristo (1920): "Exégesis del momento. Exposición Barradas en el Ateneo", La Ilustración española y americana, $\mathrm{n}^{\circ}$ 10, 15 de marzo, p. 157.

DD. AA. (2013): Barradas. Colección MNAV, cat. exp. Montevideo: Museo Nacional de Artes Visuales, pp. 18-25.

Delaunay, Robert (1957): Du cubisme à l'art abstrait, Documents inédits publiés par Pierre Francastel et suivis d'un catalogue de l'œuvre de R. Delaunay par Guy Habasque, París, SEVPEN.

Faxedas, M. Lluïsa (2015, en prensa): Del Simbolismo a la abstracción. El ideal de la unidad de las artes en los origenes del arte moderno, Trea.

Francés, José (1920): El año artístico 1919. Madrid: Mundo Latino.

Gamwell, Lynn (2002): Exploring the invisible. Art, science and the spiritual. Princeton y Oxford, Princeton University Press.

Garcia-Sedas, Pilar (1994): Joaquim Torres-Garcia i Rafael Barradas. Un diàleg escrit (1918-1928). Barcelona: Publicacions de l'Abadia de Montserrat.

Gauguin, Paul (1974): Oviri. Écrits d'un sauvage, presentados por Daniel Guérin. París: Gallimard.

Ignacios, Antonio de (1953): Historial Rafael Barradas. Montevideo.

Kupka, Frantisek (1989): La création dans les arts plastiques. París: Cercle d'Art.

Lindsay, Kenneth C. / Vergo, Peter (eds.) (1994): Kandinsky, complete writings on art. Nueva York: Da Capo Press.

Lista, Giovanni (ed.) (1973): Futurisme. Manifestes, proclamations, documents. Lausanne: L'Age d'Homme. Lista, Marcella (2006): L'œuvre d'art totale à la naissance des avant-gardes, 1908-1914. París: INHA.

Lubar, Robert S. (1991): “Art-evolució: Joaquín Torres-García y la formación social de la vanguardia en Barcelona”. En: Barradas - Torres-García, Madrid, Galería Guillermo de Osma, pp. 19-32.

Marinetti, F.T. (1996): Teoria e invenzione futurista. Milán: Mondadori.

Mas López, Jordi (2004): Josep Maria Junoy i Joan Salvat-Papasseit: dues aproximacions a l'haiku. Barcelona: Publicacions de l'Abadia de Montserrat.

Maur, Katrin von (2004): “Bach et l'art de la fugue. Modèle structurel musical pour la création d'un langage pictural abstrait”. En: Duplaix, Sophie y Lista, Marcella (dirs.), Sons \& lumières: une historie du son dans l'art du XXe siècle. París: Centro Georges Pompidou.

Morse, Jed (2006): "Art-Evolució and Vibracionismo: Torres García, Barradas, and an Art of Higher Consciousness". En: Robinson, William H., Falgàs, Jordi y Lord, Carmen Belén (dirs.), Barcelona and Modernity: Picasso, Gaudí, Dalí, New Haven y Londres: Yale University Press, pp. 333-337.

Pereda, Raquel (1989): Barradas. Montevideo: Galeria Latina.

Piñero Gil, Carmen Cecilia (2009): "Carmen Barradas: modernidad y exilio interno". En: Nagore, María, Sánchez de Andrés, Leticia y Torres, Elena (eds.). Música y cultura en la Edad de Plata, 1915-1939. Madrid: ICCMU, pp. 603-618.

Plotino (1985): Enéadas III-IV. Madrid: Gredos, (BCG, 88).

Arch. esp. arte, LXXXVIII, 351, JULIO-SEPTIEMBRE 2015, 281-298 ISSN: 0004-0428, eISSN: 1988-8511, doi: 10.3989/aearte.2015.17 
Roig, Lidia (curadora) (1992): “Joan Salvat-Papasseit: textos desconeguts”. En: Els Marges, no 46, pp. 77-86.

Roque, Georges (2002): “QQué onda? La abstracción en el arte y la ciencia”. En: Krieger Peter (ed.), Arte y ciencia. XXIV Coloquio internacional de Historia del arte. México: UNAM-IIE, pp. 169-192.

Roque, Georges (2003): “Ce grand monde des vibrations qui est à la base de l'univers”. En: DD.AA., Aux origines de l'abstraction, 1800-1914. París: Réunion des Musées Nationaux, pp. 51-67.

Roque, Georges (2009): Art et science de la couleur. Chevreul et les peintres de Delacroix à l'abstraction. París: Gallimard.

Rousseau, Pascal (dir.) (1999): Robert Delaunay, 1906-1914. De l'Impressionnisme à l'abstraction. París: MNA - Centre Georges Pompidou.

Rousseau, Pascal (2003a): “L’oeil solaire'. Une généalogie impressionniste de l'abstraction”. En: DD.AA., Aux origines de l'abstraction, 1800-1914. París: Réunion des musées nationaux, pp. 123-139.

Rousseau, Pascal (2003b): “Arabesques'. Le formalisme musical dans les débuts de l'abstraction”. En: DD.AA., Aux origines de l'abstraction, 1800-1914. París: Réunion des musées nationaux, pp. 231-245.

Rousseau, Pascal (2005): "La Galería Dalmau. La introducción de la abstracción en Catalunya y la vanguardia parisina durante la primera Guerra Mundial”. En: París - Barcelona, 1888-1937. Barcelona y París: Museu Picasso y RMN, pp. 327-337.

Rovel, Henri (1908): "Les lois d'harmonie de la Peinture et de la Musique sont les mêmes", Les Tendances Nouvelles, n. 35, marzo, pp. 721-726.

Sacs, Joan (1917): "Exposición Sergio Charchounne en los sótanos de las galerias Dalmau". La publicitat, 15 de abril, p. 8.

Salvat-Papasseit, Joan (ed.) (1918): Arc Voltaic, Barcelona.

Salvat-Papasseit, Joan (1919): Poemes en ondes hertzianes. Barcelona: Imprenta de Ramon Capera.

Santos Torroella, Rafael (1993): “Autoretrat de Barradas. Els dibuixos de Barradas a L'Esquella de la Torratxa”. En: Brihuega, Jaime y Lomba, Concha (dirs.): Barradas. Exposició antològica, 1890-1929, L'Hospitalet de Llobregat: Centre Cultural Tecla Sala, pp. 55-63.

Scriabin, Alexandre (1979): Notes et reflexions. Carnets inédites. París: Klincksieck, 1979.

Solé de Sojo, Vicenç (1917): "Films. Al marge de l'exposició Charchounne”, El poble català, 21 de abril, p. 1.

Torres-García, Joaquín (1917a): “Una vibració - un crit - un riure intel·ligent”. Un enemic del poble, n 5, agosto, p. 1.

Torres-García, Joaquín (1917b): “Los artistas uruguayos en Europa: Rafael Barradas”. El Siglo, Montevideo, 24-XI.

Torres-García, Joaquín (1984): Universalismo constructivo (2 vols.). Madrid: Alianza.

Torres-García, Joaquín (1990): Historia de mi vida. Barcelona: Paidós [1939].

Trossos (1916-1918): Barcelona.

Vallcorba Plana, Jaume (1984): “Introducció”. En: Junoy, Josep M", Obra poètica. Quaderns Crema: Barcelona, pp. XI-CXVIII.

Fecha de recepción: 18-II-2014

Fecha de aceptación: 16-VI-2014

Arch. esp. arte, LXXXVIII, 351, JULIO-SEPTIEMBRE 2015, 281-298

ISSN: 0004-0428, eISSN: 1988-8511, doi: 10.3989/aearte.2015.17 
